\title{
PETER: a torsion pendulum facility to study small forces/torques on free falling instrumented masses
}

M. Bassan ${ }^{a b}$, A. Cavalleri ${ }^{c}$, M. De Laurentis ${ }^{d e}$, F. De Marchi ${ }^{l}$, R. De Rosa ${ }^{d e}$, L. Di Fiore $^{e}$, R. Dolesi ${ }^{c}$, N. Finetti ${ }^{f i}$, F. Garufi ${ }^{\star d e}$, A. Grado ${ }^{g e}$, M. Hueller $^{c}$, L. Marconi ${ }^{j}$, L. Milano $^{d e}$, Y. Minenkov ${ }^{b}$, G. Pucacco ${ }^{a b}$, R. Stanga ${ }^{h i}$, D. Vetrugno ${ }^{c}$, M. Visco $^{k b}$, S. Vitale $^{c}$, W.J. Weber ${ }^{c}$

${ }^{a}$ Università degli Studi di Roma Tor Vergata

I-00133 Roma, Italy

${ }^{b}$ INFN - Sezione di Roma Tor Vergata

I-00133 Roma, Italy

${ }^{c}$ Dipartimento di Fisica, Università di Trento and INFN - TIFPA

I-38050 Povo (TN), Italy

${ }^{d}$ Università degli Studi di Napoli Federico II

Complesso Universitario di Monte S. Angelo, via Cintia I-80126 Napoli, Italy

e INFN Sezione di Napoli

Complesso Universitario di Monte S. Angelo, via Cintia I-80126 Napoli, Italy

${ }^{f}$ Dipartimento di Scienze Fisiche e Chimiche, Università degli Studi dellŠAquila

I-50019 I-67100 lঙ̌Aquila, Italy

${ }^{g}$ INAF-Osservatorio Astronomico di Capodimonte,

via Moiariello I-80126 Napoli, Italy

${ }^{h}$ Dipartimento di Fisica ed Astronomia, Università degli Studi di Firenze

I-50019 Firenze, Italy

${ }^{i}$ INFN - Sezione di Firenze

I-50019 Firenze, Italy

${ }^{j}$ Istituto Nazionale di Ottica - CNR

50125 Firenze, Italy

${ }^{k}$ INAF - Istituto di Astrofisica e Planetologia Spaziali

I-00133 Roma, Italy

${ }^{l}$ Dipartimento di Ingegneria Meccanica ed Aerospaziale, DIMA Università La Sapienza

I-00184 Roma, Italy

E-mail: fabio.garufiena.infn.it 
We describe here the realization and tests of a two stage torsion pendulum facility (nicknamed PETER, from Italian PEndolo Traslazionale E Rotazionale, namely translational and Rotational Pendulum) for the measurement of the Gravitational Reference Sensor (GRS) actuation Cross Talks (CT) for LISA-Pathfinder and its possible evolution. This project started within the ground testing activities for the characterization, before flight, of the GRS of LISA-Pathfinder, where it showed results consistent with what observed in flight. The apparatus could easily evolve into a facility to test small forces/torques on free falling instrumented masses, for future next generation space missions. Here, we will discuss the principle of operation of the double torsion pendulum and the initial goal of the activity, the description of the PETER apparatus, cross -talk measurement technique and results and possible extension to more than $2 \mathrm{DoF}$

GRAvitational-waves Science\&technology Symposium - GRASS2018

1-2 March 2018

Palazzo Moroni, Padova (Italy)

* Speaker. 


\section{Introduction}

Torsion pendulums are devices used in the gravitationalists community since the beginnings. Our work started within the LISA-Pathfinder (LPF) [1] project with the aim to characterise the Gravitational Reference System (GRS) [2] before flight. In this framework, starting from the initial one-stage pendulum realised in Trento [3], we developed a two stage torsion pendulum to measure the actuation cross-talk (CT) on the GRS. This device was named PETER, from the Italian PEndolo Traslazionale E Rotazionale. Here we will discuss the working principle and the measurements, as well as the possible evolution of the device as a facility.

Torsion pendulums are the instruments of choice to approach the conditions of free fall, in a ground based laboratory: this is because one degree of freedom (DoF) of the pendulum, the rotation around the suspension fiber, experiences an extremely small restoring force (better, torque) from the fiber: in pendulum jargon, this is usually called the soft, i.e. almost free, DoF.

With a single mass torsion pendulum, we can only study the effect of torques acting on the mass, while, if a 4-mass cross shaped payload is suspended to the fibre from its centre, the effects of a torque acting on a mass has the same effect as that of a small force. In order to study at the same time forces and torques on a test mass, we developed a double pendulum with two soft degrees of freedom [4], nicknamed PETER (acronym for PEndulum free in Translation and Rotation).

\section{The apparatus}

Starting from the design of the four-mass torsion pendulum, with the aim to be soft in two DoF, the choice was to hang one of the four masses to another fibre, the three remaining masses being initially kept at the same distance from the centre of the crossbar, by means of rigid legs. Thus we have a "high" fibre attached to the centre of the crossbar and a "low" fibre attached to the test mass, as shown on the left side of figure 1. The rotations around the high fibre, can be seen as translations of the test mass (in the small oscillations approximation) and the rotations are around the low fibre axis. In this way, a force on the test mass produces a translation and a torque a rotation, allowing the simultaneuous measurement of both. This allows us also to measure the actuation cross talk, i.e. rotations when actuating in force and translations when actuating torques. The whole apparatus, initially tested in Florence, was traslated to the Gravitational Physics Lab in Naples, where a concrete basement with $12 \mathrm{~m}$ long foundation poles, isolated by the rest of the building structure was prepared to host a vacuum chamber containing the pendulum.

The test mass is contained in an engineering copy of the LPF GRS with a $4 \mathrm{~mm}$ gap capacitive sensor, conditioned and read out with the same electronic device as that used in LPF. The system is equipped with a redundant readout system with combination of different sensors: Autocollimator (AC) at the crossbar level, optical read-out (ORO) at the crossbar level (added in a 2nd phase), GRS at the TM level (capacitive), ORO at the TM level.

The OROs are simple optical levers, and at the level of the TM, two levers are needed in order to disentangle translations and rotations.

The variables that are actually measured by our read-out system and the geometry of GRS sensor electrodes and actuators electrodes are shown in figure 1. The actuaton principle is also sketched in the caption of figure 1, the voltage applied to each electrode to generate a force $F$ is 


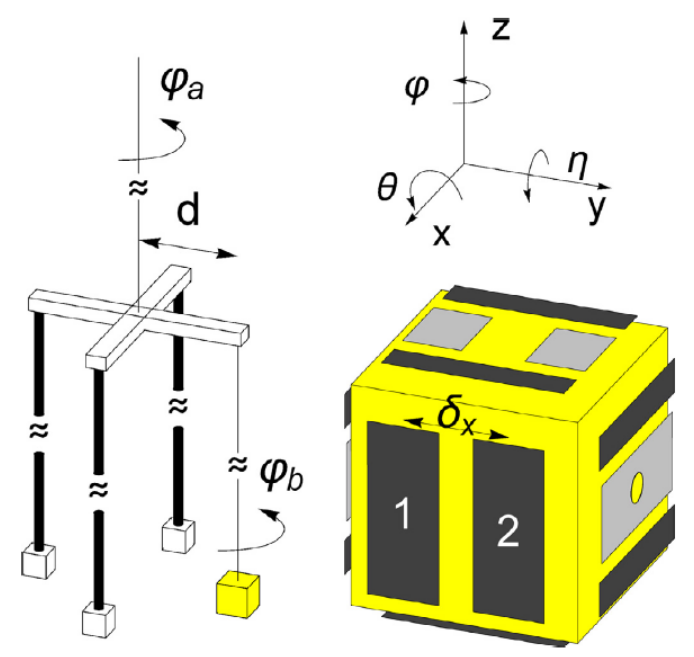

Figure 1: A sketch of the PETER pendulum, with the two torsion fibers in cascade: $\phi=\phi_{a}+\phi_{b}$ and $x=d \cdot \phi_{a}$ are the coordinates of the two soft DoFs. A diagram of the GRS electrodes, surrounding the TM, is also shown: the gray electrodes feed a.c. bias while the black ones are for sensing and actuation. Labels 1 and 2 indicate two of the four electrodes sensing $\mathrm{x}$ and $\phi$ DOFs: each one is paired in a bridge with an identical electrode on the opposite side (call them 3 and 4 respectively). By applying an electric field to electrodes 1 and 2 we can actuate a force along $\mathrm{x}$ on the TM, while electrodes 1 and 4 would be used to apply a torque on $\phi$.

propotional to the square root of $F$. The electrodes can only pull, so, one pair of electrodes is used to pull in the positive direction and the opposite pair in the negative direction.

\section{Measurements}

The measurements of translation and rotation, performed with the capacitive sensor and OROs give the same results at low frequency, but the ORO shows better sensitivity above $10 \mathrm{mHz}$, reaching the ADC noise level, as shown in figure 2.
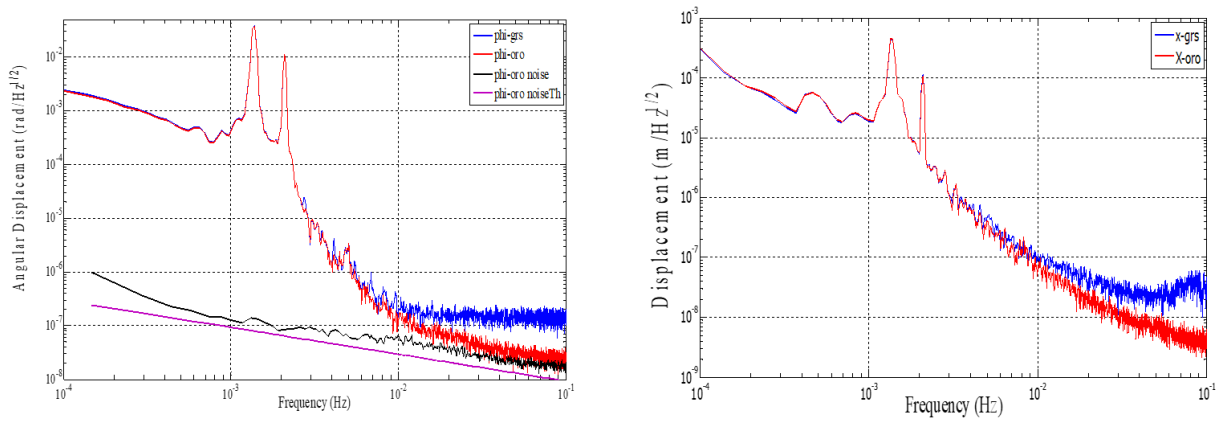

Figure 2: Measurement of rotations and translations performed with the capacitive GRS sensor (blue) and ORO (red). The theoretical and measured ADC noise is also plotted on the graphic. 
Form the time series $x(t)$ and $\phi(t)$ and their derivatives we can solve the pendulum equations of motion and compute force and torque acting on the TM [4] as shown in figure 3. The large
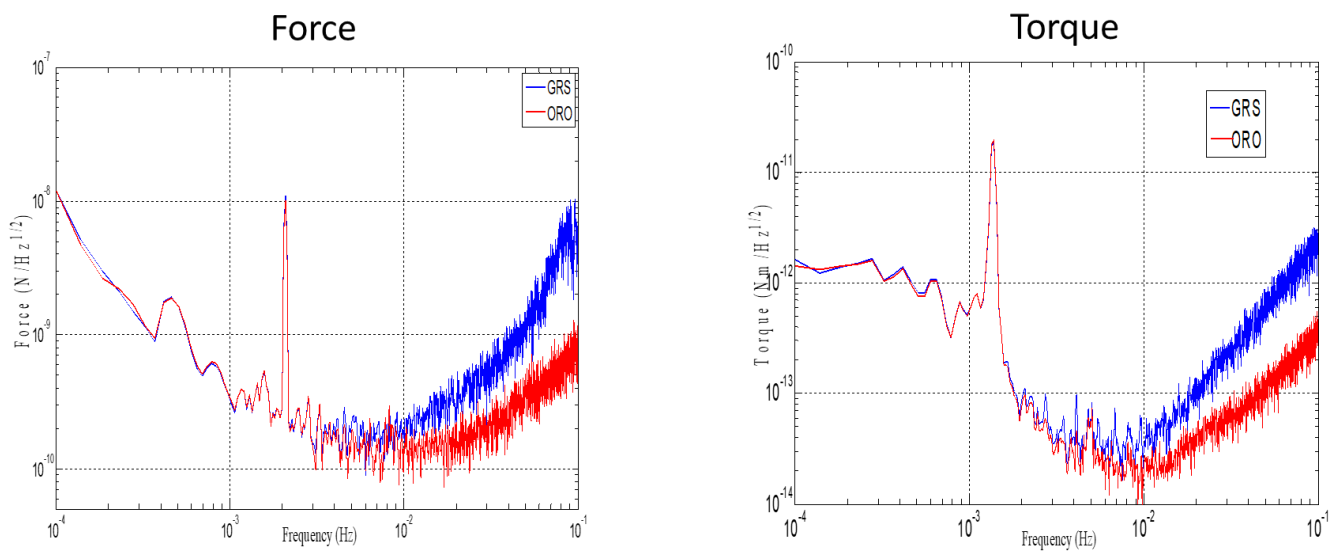

Figure 3: Measurement of force and torque performed with the capacitive GRS sensor (blue) and ORO (red) with the new pendulum version.

peak in the torque spectrum (close to $1.3 \mathrm{mHz}$ ) is due to the fact that, for a two stage pendulum, there is a frequency where a force on the TM essentially produces a rotation of the upper fiber with very little angular displacement at the TM level (a zero in the mechanical TF). This is taken into account in the equation of motion, but the presence of readout noise or other non-dynamical noise gives an overestimation of the TM rotation at this frequency, with consequent overestimation of the torque acting on the TM. The residual peak in the force spectrum is due to the fact that there are two TMs suspended with thin wires to the same crossbar arm giving (non-identical) resonant frequencies close to $2 \mathrm{mHz}$, while only one can be taken into account in the equation of motion (because there is no readout on the second TM). The remaining two suspended masses are hung more rigid wires, and do not affect the motion in this frequency band. These features, are only relevant in the frequency region around the low frequency peaks $(1.3 \mathrm{and} 2 \mathrm{mHz})$ but do not affect the CT measurements performed at higher frequencies $(14$ and $18 \mathrm{mHz})$.

By the beginning of 2017, the rigid rods holding the dummy TMs have been replaced with tungsten wires, reducing the total mass of the payload and permitting a smaller diameter upper fibre. This produced an improvement of sensitivity of about a factor 3 over the full frequency range.

By actuating a sinusoidal force on the electrodes separated by a distance $\delta x$, the force $F$ and the residual torque $\tau$ are measured. We define the contamination of torque $\tau$ when actuating in force $F$ as the force-to-torque cross-talk $C T_{F \rightarrow T}=\frac{\tau}{\delta x F}$. Similarly, the torque-to-force cross-talk $C T_{T \rightarrow F}=\frac{F \delta x}{\tau}$ can be measured by actuating a torque and measuring the residual force. We remark that, in all cases, we apply voltages to the electrodes, and measure both the resulting force and the resulting torque. The crosstalks of the above definitions are therefore estimated as ratios of measured dynamic quantities. The force exerted by each electrode can be computed as the gradient of the capacity that is in turn a function of electrode geometry and TM position (in 6 DoF). This allows us to compute force and torque as a function of position in $\mathrm{x}, \mathrm{y}$ and $\phi$, neglecting the other 3 
DoFs because they are almost constant and close to zero during the measurements. By computing the relevant quantities to the second order, we get a simple analytical model of the cross-talk to be compared to the measurements [5].

The electrode housing can be moved around the TM by step motors, allowing to measure CT as a function of the position. Each measurement must last many periods of the pendulum oscillations, so the whole measurement ( 25 steps) lasts for $50 \mathrm{~h}$ (more than two days). An example of cross talk measurement as a function of the position is shown in figure 4 .

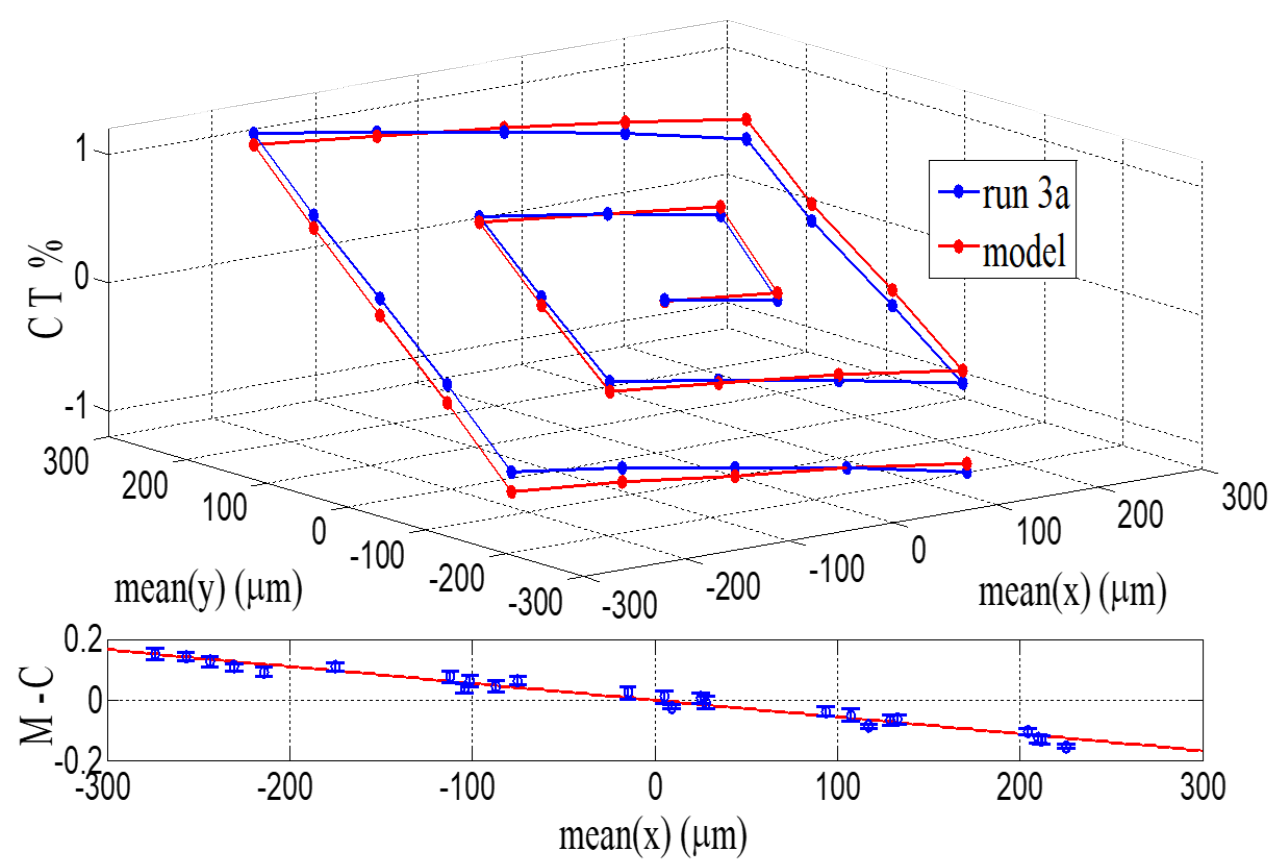

Figure 4: Crosstalk $C T_{F \rightarrow T}$ compared to the analytical model of [5] In the lower part of the figure the M-C residuals (Measured minus Computed) are shown versus the x coordinate. During the measurement, the $\phi$ variable, not shown in the plot, was changing in the range form -0.2 to $-1.25 \mathrm{mrad}$. For each experimental point, the measured value of $\mathrm{x}, \mathrm{y}$ and $\phi$ were used in the analytical model to compute the M-C residual. As we can see, there is a small discrepancy, well represented by a linear fit.

On the basis of these measurements, PETER allowed to prove that, if we look at the central region $( \pm 10 \mu \mathrm{m})$ of the GRS, that is the range of interest for the scientific operations of LPF, the measured CT is less than $0.2 \%$, in agreement with requirements. Large translation motion at low frequency and downconversion of higher frequency noise due to the 'oceanic peak' cause large pendular motion in the $0.4-1 \mathrm{~Hz}$ zone - hundreds $\mu \mathrm{m}$ instead of few $\mu m$ - because of a low efficiency of the magnetic dumper at the pre-hanger for a two stage pendulum.

This issue can be mitigated by actively controlling the suspension point in the band $0.2-1 \mathrm{~Hz}$ where the 'oceanic peak' is present. The solution is to measure ground acceleration and apply a feed-forward control to suspension point via piezo actuators in both horizontal DOFs. First experimentation of the control system are presently in course in the Naples Lab. 


\section{Possible upgrades}

Besides the control of the suspension point, the system can be ugraded to make it soft over a larger number of DoF. A possible straightforward upgrade consists in the replacement of the test mass with a further two masses torsion pendulum, in order to mesaure the forces (i.e. the translations) in two directions. Other, more complicated, schemes, including flexures at the suspension points and the hanging of the TM from a point near its centre of mass, can make the system approach the free fall conditions in more than 2 DoF although when one approaches the centre of mass, there are severe limitations due to mechanical tolerances, TM inhomogeneity and lateral accuracy in attaching the suspending wire.

\section{Conclusions}

We built and tested a two stage torsion pendulum showing a good sensitivity in torque and force mesurements. Force to torque and torque to force $\mathrm{CT}$ have been measured and are well within the LPF requirements, although force measurements are affected by higher noise than expected. Sensitivity can be improved by controlling the suspension point. First tests are ongoing in the Naples lab and show to be promising. The PETER device can be 'easily' upgraded to simulate a free falling mass to up to $6 \mathrm{DoF}$

\section{References}

[1] Antonucci F. et al, Classical and Quantum Gravity 29 (2012) 12, 124014

[2] Dolesi R. et al, al, Classical and Quantum Gravity 20 (2003) 10, S99

[3] Carbone L, et al., 6th International LISA Symposium. American Institute of Physics Conference Series vol 873 p $561-5$

[4] Bassan M. et al., Phys. Rev. Lett. 116 (2016) 051104

[5] Bassan M. et al., Astroparticle Physics 97 (2018) 19 - 26 